\title{
Experimental Study of Latent Heat Thermal Energy Storage System using PCM with Effect of Metal Configurations and Nano particles
}

\author{
Prasad Patil ${ }^{+*}$ and Tapobrata Dey ${ }^{\dagger}$ \\ †Department of Mechanical engineering, Savitribai phule pune university, D.Y. Patil college of engineering, Akurdi, Pune, India \\ Accepted 15 June 2016, Available online 20 June 2016, Special Issue-5 (June 2016)
}

\begin{abstract}
The exciting technology in present days is storage of energy in a appropriate form and which can be conservatively converted in the required form. There is mismatch in supply and demand of energy which reduces by the energy storage and also improves the reliability and performance. When the substance change the form from one phase to another due to melting or freezing the energy stored in the substance is latent heat which is one form of thermal energy. Paraffin wax is widely used for latent heat thermal energy storage system uses because of the large latent heat and also desirable thermal properties. It has lower heat transfer rate during melting and freezing processes because of its inherent low thermal conductivity. In this work solidifications and melting performance of paraffin wax dispersed with alumina (AL2O3) and copper oxide (CuO) 100 um average size in latent heat storage system is investigate experimentally.
\end{abstract}

Keywords: Phase change material, thermal energy storage, nanofluid

\section{Introduction}

Developing efficient and inexpensive energy storage devices is as important as developing new sources of energy. The thermal energy storage (TES) can be defined as the temporary storage of thermal energy at high or low temperatures. Energy storage can reduce the time or rate mismatch between energy supply and energy demand, and it plays an important role in energy conservation. Energy storage improves performance of energy systems by smoothing supply and increasing reliability. Some of the renewable energy sources can only provide energy intermittently. One of the important characteristics of a storage system is the length of time during which energy can be kept stored with acceptable losses. Another important characteristic of a storage system is its volumetric energy capacity. Therefore, a good system should have a long storage time and a small volume per unit of stored energy.

All materials are phase change materials. The most important difference between these materials is the phase change temperature. Each material makes its phase change at different temperature. In addition, each material has different value of latent heat and thermal conductivity. The main drawback of most of phase change materials is their low thermal conductivity that decreases the heat transfer rate. And hence in the present work we try to enhance heat

*Corresponding author: Prasad Patil transfer rate by insertion of high conductive metal configurations and nano-particle. The most important feature for the selected phase change material is to have its phase change temperature fitted with the application temperature range.

Nanofluids are dilute liquid suspensions of nanoparticles with at least one of their principal dimensions smaller than $100 \mathrm{~nm}$. From previous investigations, nanofluids have been found to possess enhanced thermo physical properties such as thermal conductivity, thermal diffusivity, viscosity and convective heat transfer coefficients compared to those of base fluids

\section{Literature review}

Enhancement is achieved by use of metal screens/spheres placed inside the phase change material (PCM), which is paraffin wax and results in increasing the effective thermal conductivity of the combined media of PCM and metal screens/spheres[1]. Investigated the thermal characteristics of paraffin wax with an embedded nano size copper oxide (CuO) particle. $40 \mathrm{~nm}$ mean size $\mathrm{CuO}$ particles of 2,5 and $10 \%$ by weight were dispersed in PCM for this study. The results suggested that the thermal conductivity enhances 6, 6.7 and $7.8 \%$ in liquid state (Jesumathy et al, 2004).

The performance of LHTS systems is limited by the poor thermal conductivity of PCMs employed. Various techniques reviewed are extended surfaces, employing 
multiple PCM's method, thermal conductivity enhancement and micro-encapsulation of PCM (Jegadheeswaran S. and Pohekar S.D., 2009). Investigated numerically the thermal performance enhancement of paraffin wax with $\mathrm{Al}_{2} \mathrm{O}_{3}$ and $\mathrm{CuO}$ nanoparticles. Results show that dispersing nanoparticles in smaller volumetric fractions increase the heat transfer rate (Ataer O.E., 2012).

\section{Experimental setup}

The schematic of experimental setup is shown in fig 1 . The heat storage container consists of vertical tank, in which copper tubes are inserted. PCM is filled in these tubes. The heat storage tank (container) is $750 \mathrm{~mm}$ long having inside diameter $260 \mathrm{~mm}$. The capacity of heat storage tank is 30 liters. The storage tank is insulated with glass wool of $40 \mathrm{~mm}$ thick to prevent heat loss. The copper tubes inserted into storage tank are seven in numbers and having outside diameter of $50 \mathrm{~mm}$ and thickness of $1 \mathrm{~mm}$. The length of these tubes is $680 \mathrm{~mm}$. Two stainless steel tanks of capacity 100 liters each are used for supplying water to heat storage tank. One tank supply hot water, for this two 2 KW heaters are placed inside it. Other tank is used for supplying cold water to heat storage tank.

Thermocouples are used for measuring the temperature at various points. Two thermocouples are placed in copper tubes to measure the temperature of Phase Change Material. One thermocouple is placed in each hot and cold water tank. Remaining thermocouples are used for measuring temperature of water in heat storage tank. Twelve channel temperature indicator having accuracy of one degree is used for measuring temperature. One $0.5 \mathrm{hp}$ water pump is used for supplying both hot and cold water. One flow meter having range of $1-11 \mathrm{lpm}$ is used for measuring flow of hot and cold water.

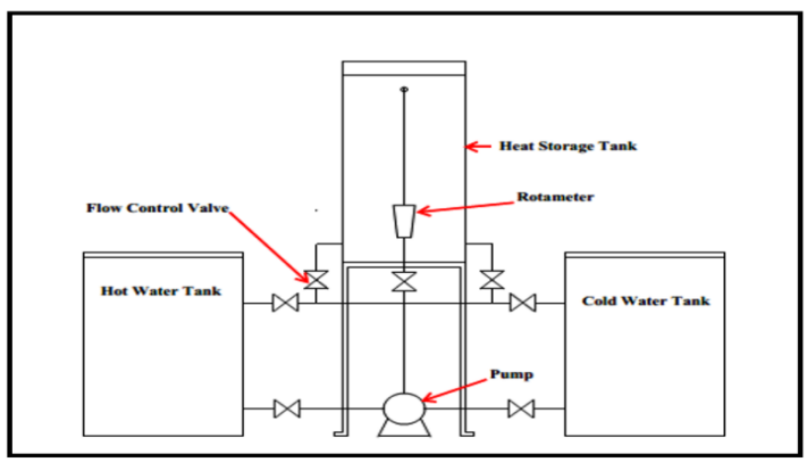

Fig.1 Schematic view of experimental setup

\section{Result and discussion}

The performance parameter computed from experimental readings was compared with melting and solidification processes of pure paraffin wax and paraffin wax with metal structures and nanomaterials. Following are the performance parameters discussed.

1) Melting and solidification processes of pure paraffin wax with variable HTF temperature.
2) Enhancement effect with insertion of copper structures in paraffin wax.

3) Enhancement effect with dispersion of $\mathrm{Al} 203$ nanoparticles at variable HTF temperature.

4) Enhancement effect with dispersion of $\mathrm{CuO}$ nanoparticles at variable HTF temperature.

5) Comparison of enhancement effect with dispersion of $\mathrm{Al} 2 \mathrm{O} 3$ with $\mathrm{CuO}$ nanomaterials.

\subsection{Melting and solidification processes of pure paraffin} wax with variable HTF temperature

After the extensive experiments the results for melting time of pure paraffin wax for various HTF temperatures (Stefan number) were plotted in the figure 2. During the initial period of the heating, the energy absorbed by the paraffin wax is in the form of sensible heat which increases the temperature of paraffin wax gradually to its melting point. Before melting begins, heat transfer through the paraffin wax is by pure conduction only. As soon as temperature rises above the melting point, the melting process of paraffin wax starts. During this the temperature increases almost linearly with time. From the figure 5.1 it is clear that the rate of charging of PCM is faster at higher value of Stefan number i.e. at $75^{\circ} \mathrm{C}$ of HTF. This happened due to large temperature difference between HTF and PCM. Time to reach melting temperature of paraffin wax is 44 minutes.

At $70^{\circ} \mathrm{C}$ HTF temperature the charging time does not reach to its melting temperature due to low heat transfer rate. From the figure 2 it is seen that upto 24 minutes, melting process of PCM is same for different Stefan number, but after that PCM temperature rapidly increases for higher value of Stefan number. The PCM temperature approaches the HTF temperature more rapidly at higher HTF temperatures. This is because of the increase in the heat transfer driving force.

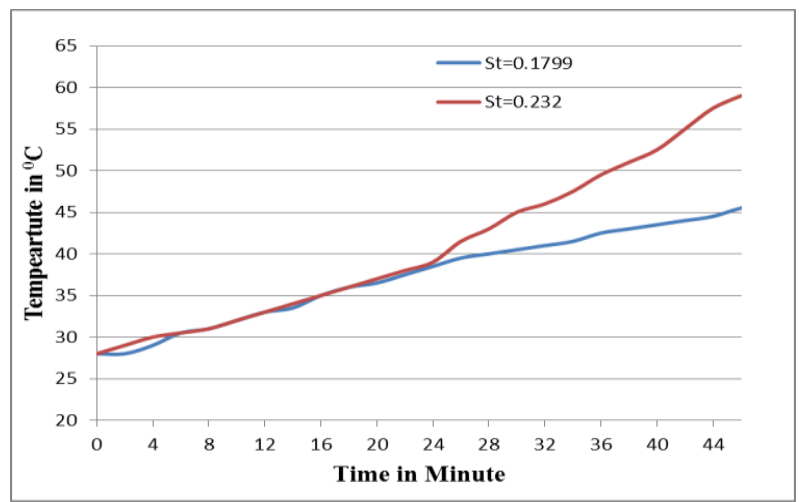

Fig. 2 Melting process of pure paraffin wax at HTF temperature 700C $(\mathrm{St}=0.1799)$ and $750 \mathrm{C}(\mathrm{St}=0.232)$

Figure 3 shows that solidification process of pure paraffin wax. Discharging or solidification experiments are performed in the same direction of the melting experiments. Immediately after charging process or melting process, the discharging experiments are initiated by passing the cold water. In essence, 
conduction heat transfer controls the heat transfer during solidification. As thermal conductivity of PCM is considerably low, the time required for solidification is expected to be longer than that of melting process

From the figure 3 it is clear that at starting upto 10 minutes temperature of paraffin wax reduces drastically, this happen due to large temperature difference between HTF and PCM. As the time passes the temperature of PCM reduces gradually and after some time it remains constant, this is due to small temperature difference between HTF and PCM.

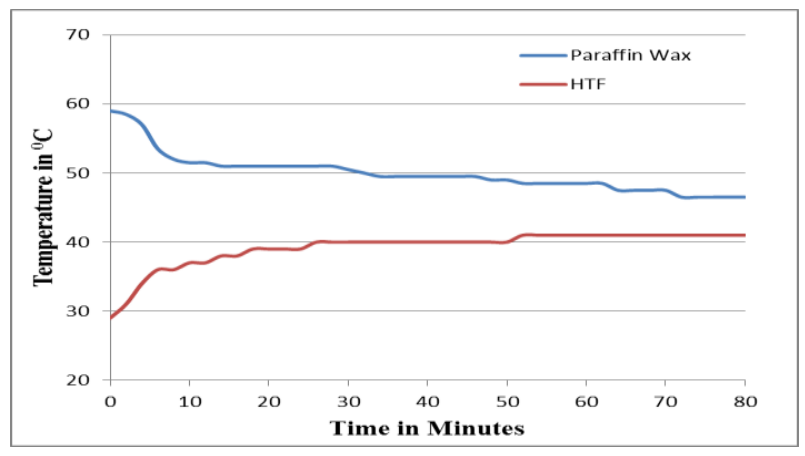

Fig. 3 Solidification process of pure paraffin wax

\subsection{Enhancement effect with insertion of copper structures in paraffin wax}

Figure 4 shows that the effect of insertion of copper strips of different structure on melting process of PCM for Stefan number 0.232. The figure shows that inserting metal strips largely reduces the charging time, charging time is reduced by $12-15$ minutes. The main reason behind this is high thermal conductivity of copper strips. The literature states that during melting heat is transferred to the PCM first by conduction and later by natural convection. Insertion of copper strips increases conduction heat transfer which results in fast melting of paraffin wax.

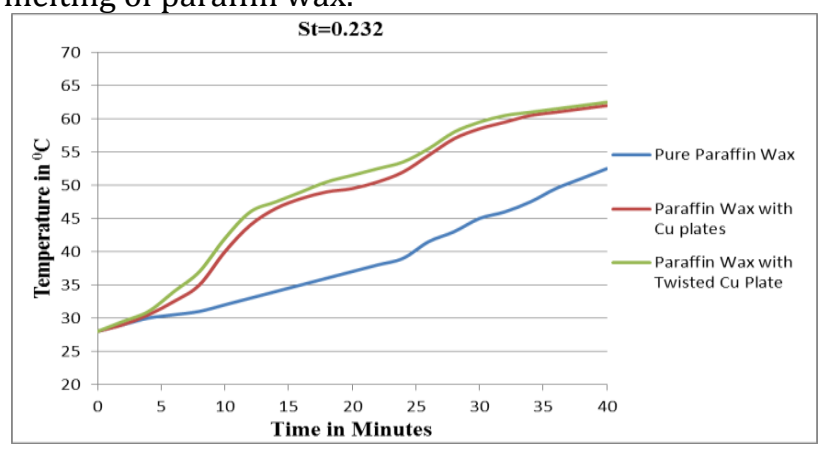

Fig. 4 Effect of insertion of copper strips on melting process of paraffin wax at Stefan number 0.232

From the figure 4 it is clear that melting time for PCM is reduces when twisted copper strips are inserted into it. The reason behind is that twisting of tubes results in more surface contact than that of simple copper strip, and we know that according to Fourier law amount of heat conducted is directly proportional to surface in contact. That's why the time required for melting of PCM is less in case of twisted tube.

Figure 5 shows that the effect of insertion of copper strips of different structure on melting process of paraffin wax for Stefan number 0.1799. Figure 5.4 shows that melting period of PCM shows that inserting metal strips reduces the charging time. For Stefan number 0.1799 (700C HTF temperature) the charging time does not reach to its melting temperature due to low heat transfer rate. But when copper strips are inserted PCM reaches to its melting temperature. The reason behind is that copper has high thermal conductivity, due to this large amount of heat is conducted into it which in turns melts the PCM faster.

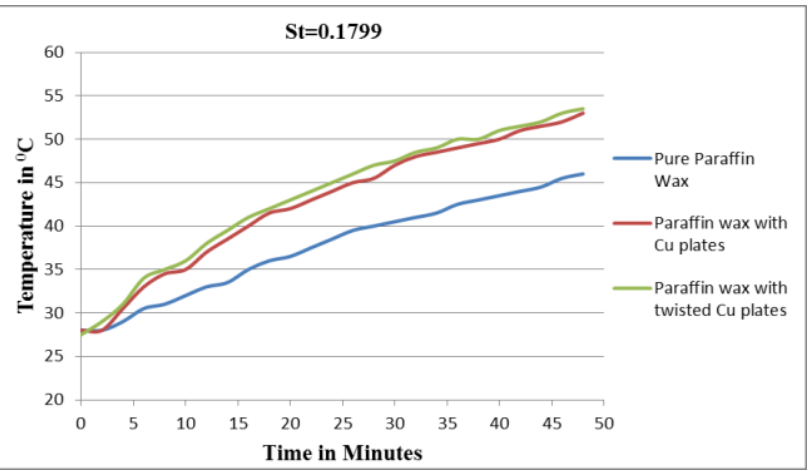

Fig.5 Effect of insertion of copper strips on melting of paraffin wax at Stefan number 0.1799

Figure 6 shows that the effect of insertion of copper strips on discharging process of paraffin wax. From figure 6 it is clear that inserting metal structure reduces the discharging time which results in fast solidification of PCM. The reason behind is that insertion of copper strips increases thermal conductivity of PCM.

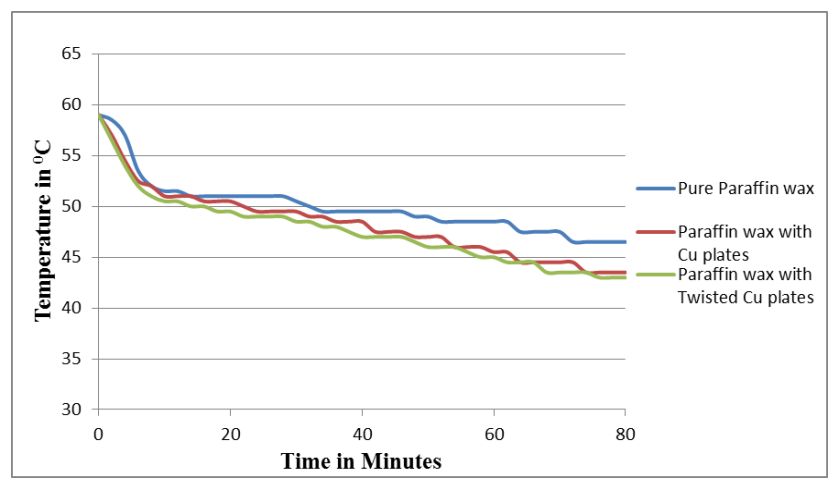

Fig.6 Effect of insertion of copper strips on solidification process of paraffin wax

\subsection{Enhancement effect with dispersion of Al203 nanoparticles at variable HTF temperature}

Figure 7 show the melting process of paraffin wax with dispersion of $\mathrm{Al} 2 \mathrm{O3}$ nanomaterial by mass concentration of $0.33 \%, 0.66 \%$ and $1.00 \%$ at Stefan number 0.232 . From figure 7 it is clear that dispersion 
of nanomaterial in PCM greatly enhance thermal conductivity of PCM, hence time required for melting is reduced as compared to pure paraffin wax. For Stefan number 0.232 melting time was reduced by 10,15 and $20 \%$ for concentration $0.33,0.66$ and 1.00 respectively. Figure 7 show the melting process of paraffin wax with dispersion of $\mathrm{Al} 2 \mathrm{O} 3$ nanomaterial by mass concentration of $0.33 \%, 0.66 \%$ and $1.00 \%$ at Stefan number 0.1799. For Stefan number 0.1799 melting time was reduced by $6.25,12.5$ and $15.6 \%$ for concentration of $0.33,0.66$ and 1.00 respectively. This results show that at high Stefan number, time required for melting is less. This happens because at high Stefan number HTF temperature is also high which results in large temperature difference between PCM and HTF and due to this large amount of heat transfer takes place between PCM and HTF. Figures shows that as the concentration of nanomaterial increases, time required for melting of PCM is reduces. The reason behind is that, nanomaterials have high thermal conductivity than PCM and due to this addition of nanomaterials increases thermal conductivity of PCM. And because of this melting time for PCM is reduces.

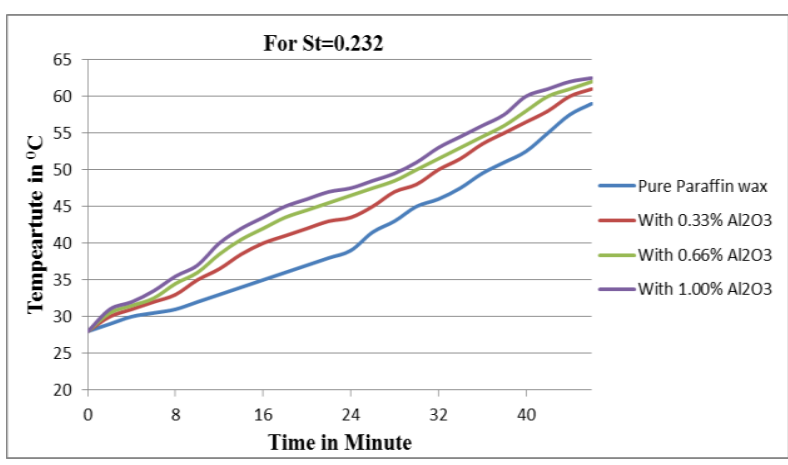

Fig. 7 Melting process of paraffin wax with dispersion of Al203 at Stefan number 0.232

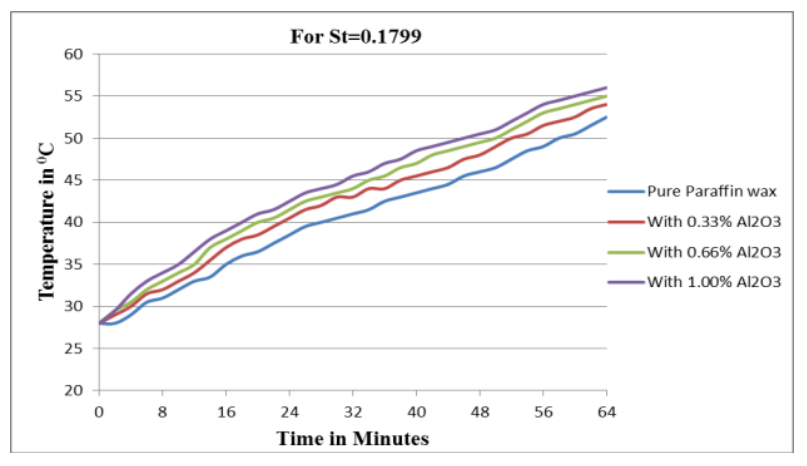

Fig. 8 Melting process of paraffin wax with dispersion of Al203 at Stefan number 0.1799

Figure 9 shows the solidification process of paraffin wax with dispersion of Al203 nanomaterial by mass concentration of $0.33,0.66$ and $1.00 \%$. From figure 5.8 it is clear that addition of nanomaterials greatly reduces solidification time of PCM. As nanomaterial concentration increases, time required for solidification is reduces. At start upto 10 minutes the graph shows drastic reduction in temperature and after that temperature of PCM gradually reduces. As nanomaterial concentration increases thermal conductivity of PCM is increases which results in decrease in time required for solidification.

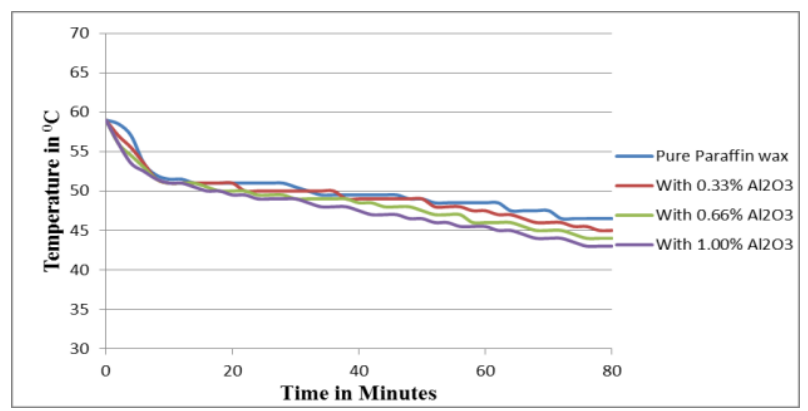

Fig. 9 Solidification process of paraffin wax with dispersion of Al203 nanomaterial

\subsection{Enhancement effect with dispersion of $\mathrm{CuO}$ nanoparticles at variable HTF temperature}

Figure 10 and 11 shows the melting process of paraffin wax with dispersion of $\mathrm{CuO}$ nanomaterial by mass concentration of $0.33,0.66$ and $1.00 \%$ at Stefan number 0.232 and 0.1799 . Figures clearly indicate that dispersion of $\mathrm{CuO}$ nanoparticles reduces time required for melting. From figures it is observed that the charging time of the paraffin wax is decreased with increasing concentration of $\mathrm{CuO}$ nanoparticles. The figures show that, for Stefan number 0.232 charging time is reduced by $6.52 \%, 10.86 \%$ and $13.0 \%$ for concentration $0.33,0.66$ and 1.00 respectively. And for Stefan number 0.1799 charging time is reduced by $3.12 \%, 6.25 \%$ and $9.3 \%$ for concentration $0.33 \%$, $0.66 \%$ and $1.00 \%$ respectively. This results show that at high value of Stefan number time required for melting is less. This happens because at high value of Stefan number HTF temperature is also high which results in large temperature difference between PCM and HTF, due to this large amount of heat transfer takes place between PCM and HTF.

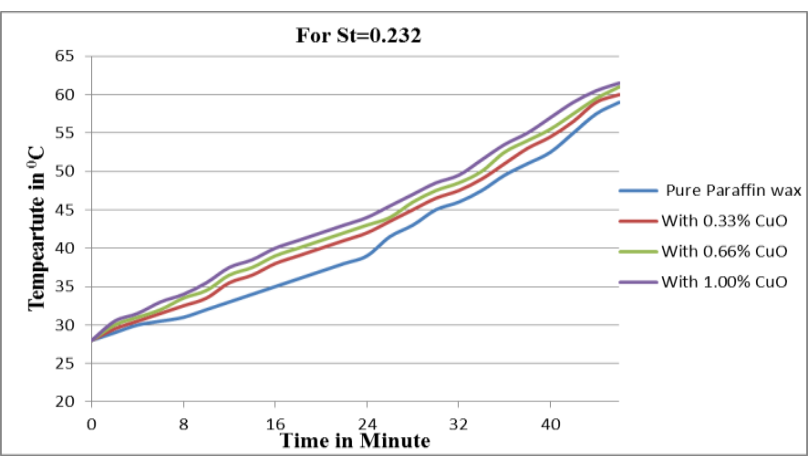

Fig.10 Melting process of paraffin wax with dispersion of $\mathrm{CuO}$ at Stefan number 0.232

Figure 12 shows the solidification process of PCM with dispersion of $\mathrm{CuO}$ nanomaterial by mass concentration 
of $0.33,0.66$ and $1.00 \%$. From figure it is clear that addition of nanomaterials greatly reduces the time required for solidification.

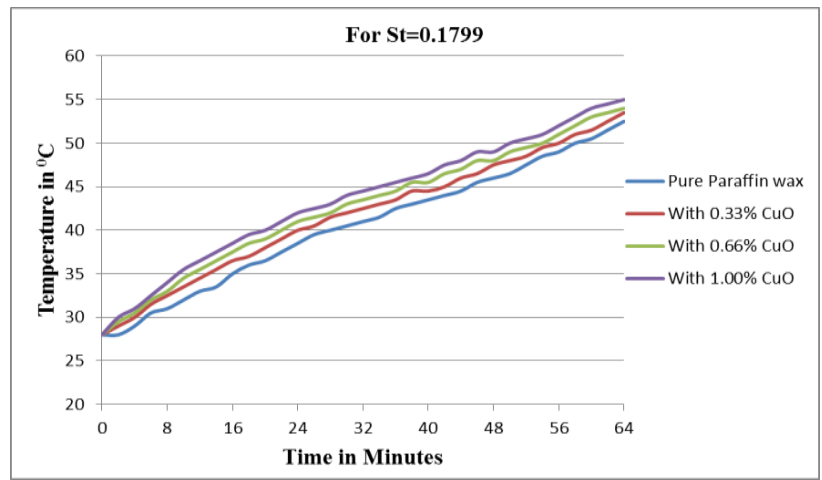

Fig. 11 Melting process of paraffin wax with dispersion of $\mathrm{CuO}$ at Stefan number 0.1799

As nanomaterial concentration increases time required for solidification reduces. At the beginning of the heat release process, the temperature of the nanoPCM decreased rapidly by transferring the sensible heat stored to the cooling water. During this period, the temperature of the nanoPCM was high, and the nanoPCM is in liquid state. This is mainly because heat transfer inside the molten nanoPCM is by natural convection and temperature gap between PCM tube and the cooling water is large. As temperature approaches the solidification temperature, the solidification process starts and proceeds into phase change controlled period. Then the nanoPCM adjacent to the PCM tube begins to freeze and discharge its latent heat. However, the frozen layer constitutes the main heat resistance for heat transfer from the interior nanoPCM to the exterior. Since the nanoPCM is basically in the liquid state, the major portion of the heat dissipated from the nano PCM is its latent heat. However towards the final period of solidification, the amount of latent heat transferred to HTF is becoming smaller and smaller, and the heat dissipation from the nanoPCM is again mainly the sensible heat of the solid paraffin wax.

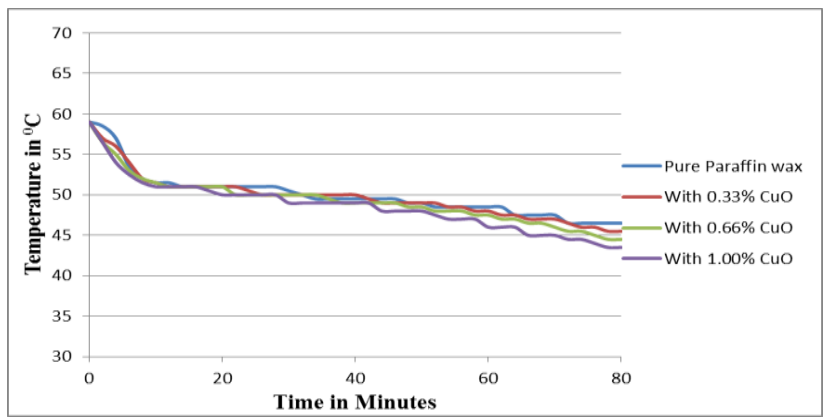

Fig. 12 Solidification process of paraffin wax with dispersion of $\mathrm{CuO}$ nanomaterial

\section{Conclusions}

1) The thermal performance of paraffin wax is greatly enhanced with inserting copper strips and twisted copper strips. Enhancement is more in case of twisted copper strips.

2) The thermal performance of paraffin wax is more at all concentration of $\mathrm{Al} 2 \mathrm{O} 3$ than $\mathrm{CuO}$.

3 ) The charge and discharge rate of thermal energy is greatly enhances when the nanomaterial concentration is increases. Dispersion of $\mathrm{Al} 2 \mathrm{O} 3$ and CuOnanomaterials in the paraffin wax enhances its low thermal conductivity and thus heat transfer rate.

4) Addition of nanoparticles does not affect the mass of PCM in LHTES so its effect on energy storage is also studied in this work

5) High rate of thermal energy storage and retrieval during the initial time period and thereafter the storage and retrieval rate of thermal energy decrease versus the time

6) In this study, the influence of some effective parameters such as mass fraction of nanoparticles and Stefan number are studied and discussed. It was observed that as nanoparticles mass fraction increases, rate of heat transfer also increases. Results in significantly reduction in the total melting and solidification time of PCM.

7) Dispersion of $\mathrm{Al} 2 \mathrm{O} 3$ in PCM by mass concentration of $0.33 \%, 0.66 \%$ and $1.00 \%$ reduces melting time approximately 10,15 and 20 minutes at HTF temperature $75^{\circ} \mathrm{C}$ and $6.52,10.86$ and 13 minutes at HTF temperature $70^{\circ} \mathrm{C}$ as compared to pure paraffin wax.

8) Dispersion of $\mathrm{CuO}$ in PCM by mass concentration of $0.33 \%, 0.66 \%$ and $1.00 \%$ reduces melting time approximately $6.25,12.5$ and 15.6 minutes at HTF temperature $75^{\circ} \mathrm{C}$ and $3.12,6.25$ and 9.3 minutes at HTF temperature $70^{\circ} \mathrm{C}$ as compared to pure paraffin wax.

9) Dispersion of Al203 in PCM by mass concentration of $0.33 \%, 0.66 \%$ and $1.00 \%$ reduces solidification time approximately 13.6, 22 and 36 minutes as compared to pure paraffin wax.

10) Dispersion of CuOin PCM by mass concentration of $0.33 \%, 0.66 \%$ and $1.00 \%$ reduces solidification time approximately 9, 15 and 22 minutes as compared to pure paraffin wax.

11) Overall, the use of nanomaterials can enhance energy charge and discharge rates only nominally. It is unlikely that this will be economically justifiable for low cost storage especially as the stability of PCMs with nanomaterials subjected to a large number of melting/solidification cycles has not been investigated yet.

\section{References}

Ettouney et al.(2012), Heat transfer enhancement by metal screens and meta spheres in phase change energy storage system

Jesumathy et al.(2004), Experimental study of enhanced heat transfer by addition of $\mathrm{CuO}$ nanoparticle

Jegadheeswaran S. and Pohekar S.D., (2009), Performance enhancement in latent heat thermal storage system: A review, Renewable and Sustainable Energy Reviews, 13, PP 2225-2244.

Ataer O.E. (2012), Storage of thermal energy storage systems, Encyclopedia of Life Support Systems.

Sharma A., Tyagi V.V., Chen C.R., and Buddhi D., (2009) Review on thermal energy storage with phase change materials and applications, Renewable and Sustainable Energy Reviews, 13, PP 318-345

Regin A. F., Solanki S.C., and Saini J.S., (2008), Heat transfer characteristics of thermal energy storage system using pcm capsules: a review, Renewable and Sustainable Energy Reviews, 12, 2438-2458.

Sharma S.D., Kitano H. and Sagara K., (2004), Phase change materials for low temperature solar thermal applications, Res. Rep. Fac. Eng. Mie Univ., 29, PP 31-64

Abhat A., (1981) Development of a modular heat exchanger with an integrated latent heat storage, Report No. BMFT FBT 81-05

Buddhi D. and Sawhney R.L., (1994), Thermal energy storage and energy conversion, School of Energy and Environmental Studies, Devi Ahilya University, Indore, India.

Farida M.M., Khudhair A.M., Razack S.A., Al-Hallaj S., (2004), A review on phase change energy storage: materials and applications, Energy Conversion and Management, 45, 1597-1615. 